\title{
Textile Products for Restaurant Business
}

\author{
Venera I. BOGDANOVA \\ Ph.D. (in Pedagogical sciences) \\ Associate Professor \\ Textile Industry Materials and Technologies Department \\ Faculty of Textile Industry Technology and Fashion \\ Kazan National Research Technological University \\ 68, Karl Marx Str., 68, Kazan, 420015, Russia \\ bogdanovavi@mail.ru \\ Guzel N. NURULLINA \\ Ph.D. (in Technical sciences) \\ Associate Professor \\ Faculty of Textile Industry Technology and Fashion \\ Textile Industry Materials and Technologies Department \\ Kazan National Research Technological University \\ 68, Karl Marx Str., 68, Kazan, 420015, Russia \\ nur.guthel@inbox.ru
}

\begin{abstract}
The article presents the results of a theoretical survey on the range of fabrics and textiles for restaurants and cafes. The study examined products for restaurant halls decoration, table linen, and staff's uniform, as well as operational requirements for this type of product. The products' environmental friendliness and fire safety of have been considered as the most important aspects of textile decoration. All textile products must comply with the technical regulations on fire safety requirements. Particular attention has been paid to the restaurant staff's uniform, emphasizing the combination of aesthetic and operational properties of products and fabrics. As special clothing for waiters, two sets of women clothes have been recommended - pantsuits and skirt suits. The features of professional fabrics with acrylic and Teflon coatings, mixed fabrics (cotton), the advantages of natural fabrics (bamboo and linen) have been considered in each category of products. In conclusion, color palettes for restaurants' or cafes' table linen and halls decor have been recommended, depending on their concept. Recommendations are based on the results of a study of psychologists on the effect of color's impact on a person's appetite.
\end{abstract}

Keywords: textiles; restaurant business; fabric; color; clothes; materials' properties.

\section{Introduction}

The restaurant business is the most demanded type of entrepreneurial activity in the modern world. Accordingly, competition is quite significant here. Despite this, many people think that it would be nice to buy a little joint and open their own restaurant or cafe with warm homely atmosphere. Each place should have its hallmark and individual identity. You do not have to spend a fortune on that, in search for something unique and unusual. A unique restaurant atmosphere can be created starting from the analysis of textiles used for its decoration, furniture, table setting, staff's uniform (Blednova, 2016). 
Restaurant textiles are an important expense for restaurant owners. But there should be no savings here, it would be detrimental for the place's reputation. Restaurant textiles include: curtains; tablecloths of various size and configurations; table napkins; envelopes (cuverts) for cutlery; placemats or toppers (table rugs for muffling the sound of dishes); summer grounds canvases; sofa cushions; covers for baskets; buffet table skirts; covers, bows, chair seats; blankets and towels. Textile products should be of excellent quality and leave only positive impressions on the visitors, since the main rule of restaurant design is the following: everything that a visitor can touch must be of the highest quality and give pleasant tactile sensations (Burenkova, 2013)

Restaurant textiles should match the eatery's atmosphere. They can be budget-friendly as well as expensive. Restaurant tablecloths, napkins and other table linen should be renewed regularly to keep up with fashion trends. Moreover, restaurant textiles should be renewed without causing too much trouble to a bar's, café's or restaurant's budget. Therefore, it is worth remembering that the cost of restaurant textiles depends primarily on the quality of materials and on the order volume. Undoubtedly, the textiles' quality and design are in the forefront when decorating a restaurant or cafe hall. Restaurant textiles reflect the level of the place and emphasize its status. More than that, properly chosen napkins, napers, chair covers and tablecloths can simplify the maintenance of excellent sanitation greatly. Textiles for the restaurant business are also called professional textiles, since they should possess a number of specific qualities in comparison to household textiles. Among them are the following:

- a large number of washes without loss of service performance;

- a persistent dye used for fabric coloring that retains its juicy color throughout the entire service life;

- dirt-water-repellent impregnation of the fabric's surface (GOST 3811-72. Textile materials).

In restaurants and cafes, it is necessary to use high quality, wear and wrinkle resistant, high-quality materials. This makes it possible to keep the eatery clean, and its business image and reputation on top. There is nothing more important than to make the restaurant's atmosphere cozy and respectable, to turn one-time visitors into regular customers by means of simple everyday things.

High-quality textiles for restaurants will help to put a special emphasis on a festive event - a banquet or a buffet table. Among a wide variety of design elements the combination of quality, decent look and reasonable price restaurant textile gives everything that can decorate the hall of any eatery and create the necessary harmony (Ivanushkina, 2012).

The choice of the set of colors for table textiles should be based on the eatery's concept. As a rule, professional dining sets have a neutral look, simple cut and minimal decoration. A naperon can be placed on the top of the main tablecloth. Its color should contrast with the tone of the latter. Naperon is designed to fulfill both decorative and practical functions. It mutes the clink of dishes, eliminates the need for the main tablecloth's frequent washing (Nurullina, 2018).

In the case of serving main dishes in restaurants and cafes, sets are used. The set is placed in front of each visitor. A plate, cutlery and glasses can be placed on top of it. The minimum size of the set is $30 \times 40 \mathrm{~cm}$. Evidence shows that oval or rectangular substitute napkins are the most practical ones. This type of table textile is made from a very dense fabric.

In the decor of some banquets, it is customary to use runners (a type of naperon that is placed across the table). Often the runner is used to create a dialogue between two people gathered at the table, as if creating a single space. The runner can be found in national cuisine serving eateries, pubs. This type of table textile looks most impressive on a table with a beautiful table top and curly legs.

Since by the word "textiles" we mean various types of fabric products, the question of the professional choice of fabrics for textile products used in the restaurant business from the point of view of aesthetic and operational characteristics aroused interest. 


\section{Materials and Methods}

General logical methods and techniques for studying textile materials and products in the restaurant business have been used within the framework of the article's topic.

Textile materials used in restaurant halls interior have an important aesthetic role. Furnishing fabrics of high density are most often used in the hall decoration.

The operational load of decorative textiles for restaurants is much higher than that of home textiles, they have to be washed and cleaned much more often. In restaurants dry cleaning of blankets and sachets from armchairs or sofas, washing of tablecloths, towels and napkins is done after each visitor. Curtains are usually washed 2-3 times a month. Therefore, these items are made from special materials called professional fabrics. Such fabrics should meet the following requirements: high dimensional stability after repeated washings, easy cleaning and wet heat treatment; the presence of moisture and dirt-repellent impregnation; the minimum rate of accumulation of static electricity and fire safety (Beninate et. al., 1979).

Professional fabrics for restaurants are distinguished by a variety of textures. "Blackout" is among the most popular ones because of its high opacity index (Besshaposhnikova et. al., 2013). It is used primarily for the manufacture of curtains and decorative veils. Two- and three-layer jacquard fabrics with a dirt-repellent impregnation are well suited for bedspreads. The material consists of two layers: the front one made of natural fibers and the foam base. Silk and satin blankets also look very aesthetically pleasing, but they are much more expensive than jacquard ones.

Canvas is called a new generation fabric (Ma et. al., 1997). It combines all properties that a highquality fabric should have: wearing qualities, strength, durability, aesthetic look. Canvas is made from cotton and synthetics and has two layers. The top layer consists mainly of polyester (85\%) and nylon, the base is made of polyester (65\%) and cotton or linen. It acquires resistance to wear and tear, high strength thanks to special technology weaving. The following qualities of canvas can be named as its undeniable advantages: durability, reliability, shape and color stability, tactile pleasantness, breathability, ease of care. Covers for upholstered furniture, durable covers, and exquisite curtains are made from canvas.

Chanselise is often used for curtains. It is a double-sided satin plain fabric with sheen, very soft and smooth in feel. Upon closer examination, the fabric shows stripes-strokes with the base colour along its structure. Chanselize is a fabric made of artificial components, it consists mainly of polyester (100\%), therefore it has good operational properties, is affordable and available in a variety of colors. Products made from such fabric have a strong, dense texture that blocks the sunlight perfectly. The material is practical, easy to clean and retains its shape well after numerous washes.

Velvet is called the fabric of kings. It is truly unique. It suits perfectly for making luxury goods, comfort clothing, exquisite home textiles, luxury upholstery. The procedure of velvet fabric production has its own subtleties. It can be divided into the following stages: weaving, dyeing, finishing. Initially, the canvas is made in two layers, "fastened" with pile velvet. It is subsequently split on a lathe. Each production stage is strictly controlled, and finished products are checked for compliance with international standards. The unique advantages of the fabric are the following: strength; wear resistance; heat saving; hypoallergenic; breathability; aesthetics (beautiful shine), softness; elasticity (inherent in stretch velvet); hygroscopicity (absorbs moisture perfectly) (Besshaposhnikova, 2013).

Recently, bamboo fiber products have become widespread. This material takes pride of place among professional fabrics due to its high antibacterial properties and excellent wear resistance.

Another important parameter when choosing accessories for the hall's interior is density. The minimum allowable density of a sofa cushion is $600-750 \mathrm{~g} / \mathrm{sq} . \mathrm{m}$, and the maximum is $1000 \mathrm{~g} / \mathrm{sq}$. $\mathrm{m}$. Accordingly the height of a cushion depends on its density. The usage of artificial materials is 
allowed in the design of the restaurant hall if they comply with the technical regulations on fire safety requirements (Federal Law N 123 dated 22.07.2008).

Materials' fire safety is achieved in two ways: using hardly flammable fibers in the content of the fabric and applying fire retardant impregnation. Refractory-impregnated fabrics are more expensive to operate, since dirt is removed from them only by dry cleaning. During normal washing, the impregnation is washed off and the material loses its flame retardant properties. An example of a synthetic flame retardant fiber is "trevira" - a polyester with tphosphorus molecules embedded in its structure. This is a patented development of the German brand Trevira Gmbh, which at one time became a big breakthrough in the field of professional textiles.

Another criterion for assessing the quality of decorative fabrics for restaurants is their environmental friendliness. The norms for the fabric's assessment for the content of harmful substances (plasticizers, formaldehydes, etc.) are regulated by the international OEKO-TEX ${ }^{\circledR}$ standard (Tukhanova et. al., 2017). If the fabric meets its requirements, the manufacturer gets the right to place the OEKO-TEX ${ }^{\circledR}$ trademark on the product label. The presence of such a sign indicates that the fabric is of high quality and environmentally friendly.

\section{Results and Discussion}

Fabrics designed for table linen are different in purpose: for tablecloths, napkins, napers, chair covers, buffet aprons, paths, placemats. Restaurant table linen should have a long service life, stain removal, color retention and good hygroscopic properties. In most cases cotton or linen fabrics as well as blended fabrics in the ratio of $50 \%$ cotton and $50 \%$ polyester are used as tablecloths in restaurants (Burenkova, 2013; GOST 3811-72).

The advantages of cotton fabric are the following: resistance to burning, easy moisture absorption, pleasantness to the touch, high dimensional stability during a significant number of washes. The disadvantages include the ability to wrinkle of the fabrics in this assortment group. This disadvantage is offset by the fact that cotton fabrics are wet-heat treated easily.

Blended fabrics made from cotton in combination with polyester have the following advantages: resistance to pill formation; slight changes in linear dimensions and tendency to crease; good drapability rate and removal of various dirt types from the fabric surface.

Tablecloths, napkins, runners, naperons are the basis of table setting and have three main functions: aesthetic, reducing the cutlery sliding on the table surface, muffling the clatter of plates and cutlery against the table top. The surface density of the fabric for the tablecloth must be at least $190 \mathrm{~g} / \mathrm{m}^{2}$. Currently, in addition to the above mentioned, fabrics with various coatings (acrylic, Teflon) are widely used.

Acrylic coated tablecloth fabrics are highly wear, water and high temperatures resistant. Hot dishes can be put on the tablecloths made of acrylic coated fabrics, as they can withstand high temperatures. The advantages of the fabric also include the fact that the seamy surface does not slip, the acrylic layer of the outer side gives the fabrics oil-grease-repellent properties. Solvents do not affect the impregnation of the fabric. A significant disadvantage is the quality characteristics deterioration in the course of use.

In addition to the above mentioned, table linen fabrics with Teflon coating are currently widely used. This group of fabrics has a similar disadvantage - deterioration of the impregnation properties during operation. In the process table linen usage, the resistance of oil-dirt-water-repellent qualities deteriorates.

Natural fabrics flax in particular are the most prestigious ones. From the point of view of weaving the first place is undoubtedly occupied by jacquard fabrics. Despite the respectable look of the tableware, this category has a number of disadvantages: it poorly not lends itself to wet heat 
treatment; there is a significant change in linear dimensions after washing; it is not durable. In this regard, the fabric consumption when sewing table linen increases, and as a result, material costs also increase. Despite the above-mentioned disadvantages, prestigious restaurants prefer highquality linen tablecloths and napkins. The solution to these disadvantages at the moment is the process of linen "softening". Stone wash and enzyme wash are special ways of flax and products made from it treatment, that are rapidly gaining popularity. More than $90 \%$ of flax products sold in Europe have undergone this kind of treatment. It is not difficult to distinguish such products since they have characteristic "tightness", the fabric is soft and flowing, despite its high density. Specialized equipment (industrial washing machine) is used to soften fabrics or finished products. Methods of enzyme softening and washing with silicones are used. After enzyme washing, the product may change color (the color becomes less bright, lightens), and shrink up to 30\%. Fabrics are softened with cuts and the length of one cut is no more than 5-7 meters. The minimum volume for softening is 20 meters in total area.

Staff's uniform is an important textile item in restaurant business. The service personnel look is an important image component of such service establishments as restaurants or cafes. A receptionist at the counter wearing a stylish uniform, an elegantly dressed head waiter is always a positive sign of the place. Cooks and waiters in clean and beautiful costumes always inspire the customers' trust. Clothing for service personnel is primarily clothing for work therefore, it should be comfortable. A beautiful and stylish waiters' uniform should be made in such a way to be convenient for employees to carry out their daily duties, not restricting their movements, while looking beautiful on the figure.

Modern waiters can wear dress, skirt, or trousers. More and more restaurant executives are inclined to let the staff have both a trouser set and a skirt with a blouse or dress. All these uniform items for the staff working in one restaurant are created in the same style, are interchangeable and let the staff carry out professional duties without any problems.

Recently an apron has been an integral part of a waiter's clothing. It was a small lace apron tied at the waist and covering the belly. The functional component of such a detail of the waiter was insignificant, with the exception of hiding the tips. However, the apron was worn throughout the shift and played the role of a kind of visiting card.

The modern apron is more practical: it is an independent piece of clothing of different styles: a pinafore, a double-sided cape with ribbons at the waist or in the form of a tunic. All varieties have pockets for keys and small items. It is worn over the suit to prevent staining and is made from easily washable materials.

The next stage of our research was to analyze the color category of textiles for the restaurant business.

The influence of colors on the people's mood and the human body, appetite, has been studied by psychologists (Burenkova, 2013; Ivanushkina, 2012). The below mentioned colours were classified as appetite-stimulating ones. Red colour increases blood pressure and pulse, and also stimulates appetite. Therefore, in many restaurants, tables are covered with red tablecloths. Orange colour stimulates the brain, which leads to increased mental alertness and the feeling of hunger as a result of it. Yellow is a very cheerful colour. Happy and energetic people have better appetite than sad or calm ones. Green is good for maintaining a healthy diet. Turquoise is associated with feelings of happiness and carelessness and can whet your appetite.

Coloured tablecloths are suitable for informal events. Such table setting is practical, bright saturated colours cheer up and adds charm to the feast. When choosing coloured linen, it is necessary to combine it with dishes. An important condition for the tablecloth and napkins set is their combination in color and fabric. A prerequisite for completeness is a combination of table linen by colour. It should be borne in mind that it is advisable to use for napkins fabric with a lower surface 
density, but the same texture and composition. If the fabric is the same in terms of density, the colours should be different.

White remains classic and trendy colour for a long time. This is due to the fact that snow-white tablecloths and napkins are appropriate in any setting and are combined with any colour scheme.

Blue is associated with calmness. People in a calm, relaxed condition are more likely to take naps rather than eat. Probably this colour suppresses appetite. Violet shades rarely cause appetite (Nurullina, 2018).

The analysis of the textures used for the restaurant business showed that the most widely used is the vegetable print - the basic classic. First of all, this is due to the fact that no special decoration techniques are required when setting the table. In the category of plain linen, the first positions are taken by models of champagne or tea rose shades that allow you to create an atmosphere of sophistication at a celebration. For interior decoration and table linen, it is more advisable to use fabrics of warm colors. Cold shades have a calming effect on the human psyche; therefore, they are used in the restaurant business to a limited extent.

\section{Conclusion}

In the conclusion of the study, it should be understood that when choosing a fabric, there is a direct relationship between price and quality. The higher the price is, the better is the product, and therefore the longer its service life is going to be. Much depends on the care, that is, on the work of the laundry company.

Restaurants are recommended to give preference to the golden mean. The price of the products should not hit the budget of the enterprise too much, but at the same time, the operational properties of the material should be taken into account. Professionals also advise working with one textile accessories manufacturer.

It is also necessary to say a few words about the drapery of restaurant halls. This concept includes curtains, bedspreads for chairs and sofas, decorative cushions. Usually they are all selected, if not from the same material, then at least in one color scheme. A prerequisite for all these interior items is the presence of an anti-flammable impregnation.

Blended fabrics are the most popular ones, due to their raw material composition, consisting of polyester threads, viscose or cotton yarn. They have a number of advantages: they are resistant to deformation, not subjected to changes in linear dimensions during washing, and dirt of various nature can be easily removed from the surface.

From the point of view of operation, it is more profitable to purchase linen from blended fabrics, since they have higher wear resistance rates than cotton, and are also cheaper.

The restaurants' textile equipment should be approached responsibly. Poor quality materials and tasteless design can ruin the experience of high-quality service. Practicality, aesthetics, durability are the three pillars on which a reasonable choice of decorative textiles, special clothing and table linen for a restaurant rests.

\section{References}

Beninate, J.V., Morean J.P. (1979). Journal of Fire-Retardant Chemistry, 6, 193-205.

Besshaposhnikova, V.I. (2013). Features of fire-protection of textiles through the action of laser radiation. Fibre Chemistry, 44(6), 347-351.

Besshaposhnikova, V.I., Zagoruiko, M.V., Pulina, K.I. (2013). Method for fire protection of woolcontaining cloths for special clothing. Fibre Chemistry, 44(6), 368-371. 
Blednova, T.V. (2016). Requirements for the presence of special clothes and uniforms in restaurant business. Territory of Science, 3, 174-179.

Burenkova, O.A. (2013). The influence of color on the psychophysiological state of the individual. Successes of modern natural science, 10, 153-154.

GOST 3811-72. Textile materials. Fabrics, non-woven fabrics and items. Methods for determining linear dimensions, linear and surface densities.

Ivanushkina, I.Yu. (2012). Influence of color and light on a person. Biomedical Engineering and Electronics, 2, 75-77.

Ma, Z., Zhao, W., Liu, Y., Shi, J. (1997). Journal of Applied Polymer Science, 63, 1511-1515.

Nurullina, G.N. (2018). Ensuring the quality of services performance at service enterprises. 5th International Youth Scientific and Practical Conference. Product quality: control, management, enhancement, planning. Digest of articles, 98-101.

Tukhanova V.Yu., Tikhonova T.P. (2017). Engineering confectioning of materials for garments. Modern problems of science and education, 1, 55-59. 